The cheerleader effect is robust to experimental manipulations of presentation time

Daniel J. Carragher ${ }^{1,2 *}$, Nicole A. Thomas ${ }^{1,3}$, O. Scott Gwinn ${ }^{1} \&$ Michael E. R. Nicholls ${ }^{1}$

${ }^{1}$ College of Education, Psychology, and Social Work

Flinders University

Adelaide, Australia

${ }^{2}$ Faculty of Natural Sciences

University of Stirling

Scotland, United Kingdom

${ }^{3}$ Turner Institute for Brain and Mental Health

Monash University

Melbourne, Australia

** This is an Accepted Manuscript of an article published by Taylor \& Francis Grqup in Journal of Cognitive Psychology on 05 Jun 2020, available online: http:// www.tandfonline.com/10.1080/20445911.2020.1776718 $*$

Total Word Count: 5000 approx.

*Corresponding Author:

Daniel J. Carragher

Faculty of Natural Sciences

University of Stirling

Stirling, UK, FK9 4LA

danieljcarragher@gmail.com 


\begin{abstract}
The "cheerleader effect" occurs when the same face is perceived to be significantly more attractive when seen among a group of faces compared to alone. Since perceived attractiveness decreases with additional viewing time, we investigated whether the cheerleader effect occurs simply because the target face is seen for less time in a group than it is alone. Observers rated the attractiveness of each target face twice; once in a group, and once alone. We manipulated the amount of time that each group image was presented for prior to the cue toward the target face $(300,1000,2000,3000$, or 7000 milliseconds). Faces were perceived to be significantly more attractive in each group condition, regardless of presentation time, replicating the cheerleader effect. Furthermore, uncued presentation time did not modulate the magnitude of this increase, demonstrating that a presentation time discrepancy does not contribute to the size of the typical cheerleader effect.
\end{abstract}

Keywords: facial attractiveness; social perception; first impressions; ensemble perception 


\section{The cheerleader effect is robust to experimental manipulations of presentation time}

A growing number of studies provide evidence for the "cheerleader effect", a phenomenon whereby an observer perceives the same face to be significantly more attractive when seen among a group of other faces, compared to when it is seen alone (Carragher, 2018; Carragher, Lawrence, Thomas, \& Nicholls, 2018; Carragher, Thomas, Gwinn, \& Nicholls, 2019; McDowell \& Starratt, 2019; Walker \& Vul, 2014; Ying, Burns, Lin, \& Xu, 2019). Found to occur for both male and female faces (Walker \& Vul, 2014), the cheerleader effect results in an average increase in attractiveness of approximately $1.5-2.0 \%(d=0.60-0.70)$ for a face that is seen in a group (Carragher, 2018).

Despite strong evidence that the cheerleader effect is a robust and reliable phenomenon, the cause of the effect has not yet been established. Walker and Vul (2014) initially proposed that the cheerleader effect was the consequence of a complex hierarchical encoding process. First, upon viewing a scene with multiple elements, the visual system quickly extracts accurate summary statistics in the form of an ensemble average (Ariely, 2001; Whitney \& Yamanashi Leib, 2018). This process of ensemble coding applies to the perception of faces, such that observers can quickly extract the average expression (Griffiths, Rhodes, Jeffery, Palermo, \& Neumann, 2018; Haberman \& Whitney, 2007, 2009) or identity shown among a group of faces (de Fockert \& Wolfenstein, 2009; Neumann, Schweinberger, \& Burton, 2013). Second, faces with average characteristics are often perceived to be highly attractive (Rhodes, 2006), such that digitally averaged faces are frequently found to be more attractive than each of the individual faces used in their creation (Carragher et al., 2019; Langlois \& Roggman, 1990). Finally, when asked to recall the characteristics of an individual item from a group, observers display a bias to recall the item as having been more alike the ensemble average than it truly was (Griffiths et al., 2018), suggesting that visual working memory has a hierarchical structure wherein knowledge about individual items is stored in 
reference to the summary statistics of the group (Brady \& Alvarez, 2011; Lew \& Vul, 2015). Putting these processes together, Walker and Vul (2014) proposed that the cheerleader effect occurs because observers systematically recall an individual face from a group as having been more alike the highly attractive ensemble average than it really was.

Despite proposing the hierarchical encoding mechanism, Walker and Vul (2014) did not explicitly test whether the cheerleader effect was caused by such a process. Recent investigations by Carragher et al. (2019) and Ying et al. (2019) have examined what role, if any, hierarchical encoding has in the cheerleader effect (also described as the "friend effect" by Ying and colleagues). Carragher et al. (2019) found that the cheerleader effect was reduced, but remained statistically significant, in group conditions that were incompatible with hierarchical encoding, including when the target face was shown in groups with nonhuman distractor images. Similarly, Ying et al. (2019) reported that although a statistically significant cheerleader effect occurred in each group condition tested, the size of the effect was modulated by the attractiveness of the other faces in the group; being seen in an unattractive group led to a larger increase in attractiveness for a face than being seen in an attractive group. In a related paradigm, van Osch, Blanken, Meijs, and van Wolferen (2015) also reported that the attractiveness of the face being evaluated modulated the size of the cheerleader effect, though processes of contrast and assimilation (Wedell, Parducci, \& Geiselman, 1987). Taken together, these findings are inconsistent with the notion that the hierarchical encoding mechanism is the only cause of the cheerleader effect (Carragher et al., 2019; Ying et al., 2019).

Instead, Carragher et al. (2019) and Ying et al. (2019) separately suggested that the cheerleader effect might be the result of two separate mechanisms, which together cause a face to appear more attractive in a group. Noting that some increase in attractiveness appears to occur regardless of the characteristics of the other images in the group, both authors 
proposed that this initial increase in attractiveness might occur through a type of social inference mechanism (Carragher et al., 2019; Ying et al., 2019). Being seen in a group might cause the observer to infer that the target face is desirable, because they appear to have been endorsed by the other members of the group (Carragher, 2018; Carragher et al., 2019; Rodeheffer, Proffitt Leyva, \& Hill, 2016). Ying et al. (2019) suggested that the initial increase in attractiveness is then further modulated by a second mechanism of contrast, which is determined by the relative attractiveness of the other faces in the group compared to the target face. On the other hand, Carragher et al. (2019) are more equivocal about the nature of the possible second mechanism, but ultimately suggest that if a social inference mechanism does cause an initial increase in attractiveness, then the additional variance in the size of the effect might still be consistent with the previously proposed hierarchical encoding mechanism (Walker \& Vul, 2014).

Although it is possible that two separate mechanisms contribute to the cheerleader effect (Carragher et al., 2019; Ying et al., 2019), an alternative possibility is that a known methodological confound might inadvertently contribute to the size of the typical cheerleader effect (Carragher, 2018). The experiments reported by Carragher and colleagues $(2018 ; 2019)$ have used the same trial procedure that Walker and Vul introduced to measure the cheerleader effect (Experiment 4; 2014). In this paradigm, the same target face is presented twice; once in a group with two distractor faces, and once alone as a portrait. During group trials, the group image is initially presented for $2000 \mathrm{~ms}$ without the target face identified (uncued presentation), and the observer is free to gaze at the 3 faces in the group. The target face is then cued in the group for $1000 \mathrm{~ms}$, before all faces are removed from the display and the observer makes an attractiveness rating for the target face. Assuming that the observer disperses their gaze approximately equally across the 3 faces in the group during the uncued presentation, and then exclusively fixates the target face once cued, the target face is viewed 
for approximately $1667 \mathrm{~ms}$ during a group trial [(2000 ms / 3 faces $)+1000 \mathrm{~ms}=1667 \mathrm{~ms}]$. However, the same target face is presented for $2000 \mathrm{~ms}$ in the alone presentation condition.

It is this discrepancy between the presentation time of the target face in the group and alone conditions that might inadvertently contribute to the size of the cheerleader effect, giving the appearance of two separate mechanisms when there is only one (Carragher, 2018). Previous research has shown that increased viewing time decreases the perceived liking of faces (Gerger, Forster, \& Leder, 2017). Crucially, additional viewing time also leads to decreases in attractiveness ratings (Willis \& Todorov, 2006), as the same face is perceived to be less attractive after being seen for $1000 \mathrm{~ms}$ compared to $20 \mathrm{~ms}$ (Saegusa \& Watanabe, 2016), or when viewed for 5000 ms compared to 200 ms (Rashidi, Pazhoohi, \& Hosseinchari, 2012). Taken together, these findings raise the possibility that the size of the cheerleader effects reported using the conventional paradigm might be inflated simply because the target face is seen for less time in a group than it is alone. To test this possibility, we investigated whether the uncued presentation time of the group image influences the size of the cheerleader effect.

The idea that viewing time might contribute to the cheerleader effect was previously considered by Walker and Vul (Experiment 3; 2014), who reported that reducing the presentation time of the alone condition to match the viewing time possible in the group condition did not influence the size of the cheerleader effect. However, Walker and Vul (2014) presented each group image three times so that a cheerleader effect measure could be collected for each face in the group. Because each group image was presented more than once, it is possible that observers altered their gaze across group images that had already been seen, to avoid fixating a face that had already been identified as a target (knowing that it would not be the target again). Consequently, the amount of time that each target face was fixated could have been influenced by the number of times that the same group image had 
been presented previously, potentially limiting the conclusions that can be drawn from this study (Walker \& Vul, 2014). The current study overcomes this limitation by presenting each group image once, thereby reducing any motivation for observers to distribute their gaze unevenly across the faces in the group.

In the current study, we employed a between-participants design to investigate the influence of presentation time on the size of the cheerleader effect. In Experiment 1a, all participants experienced moderate changes to the uncued presentation time of the group image, which were 1000 ms, 2000 ms (control condition; Carragher et al., 2018; Carragher et al., 2019; Walker \& Vul, 2014), and 3000 ms. The 3000 ms condition was specifically designed to resolve the presentation time discrepancy between the control condition and the alone condition described above (Carragher, 2018; Walker \& Vul, 2014). Crucially, if observers distribute their gaze evenly across the 3 faces in the group during the uncued 3000 ms presentation (1000 ms per face), and then exclusively fixate the target face when cued $(1000 \mathrm{~ms})$, the target face is seen for the same amount of time as it is in the alone condition (2000 ms). Therefore, a significantly smaller cheerleader effect in the $3000 \mathrm{~ms}$ condition compared to the control condition $(2000 \mathrm{~ms})$ would indicate that the discrepancy between the presentation duration of the group image and the alone image in the existing paradigm contributes to the size of the cheerleader effect.

To further explore whether any change to the uncued presentation duration of the group image would modulate the size of the cheerleader effect, participants in Experiment $1 \mathrm{~b}$ experienced extreme changes to the uncued presentation time of the group image. For these participants, the shorter presentation time was further decreased to $300 \mathrm{~ms}$, while the longer presentation time was increased to $7000 \mathrm{~ms}$. We selected $300 \mathrm{~ms}$ as the shortest uncued presentation time to allow participants the opportunity for saccadic eye movements across the group image, increasing the possibility that each face could be briefly examined (Crouzet, 
Kirchner, \& Thorpe, 2010; Rayner, 1998). We selected $7000 \mathrm{~ms}$ as the longest presentation time because, assuming equal gaze across the group image, observers could potentially fixate the target face for $3334 \mathrm{~ms}$, which is twice the amount of time possible in the established control condition.

In keeping with previous reports that attractiveness ratings decrease as observers are given more time to examine a face (Rashidi et al., 2012; Saegusa \& Watanabe, 2016; Willis $\&$ Todorov, 2006), we expected a negative relationship between presentation time and the cheerleader effect. First, we predicted that if the methodological presentation time discrepancy contributes to the cheerleader effect, then the cheerleader effect should be larger in the 2000 ms control condition than the 3000 ms condition in Experiment 1a. Second, we predicted that the largest cheerleader effects would occur for the shortest uncued group presentations and decrease as presentation time increased.

\section{Method}

\section{Participants}

We have previously shown that the cheerleader effect occurs with a medium effect size ( $d=0.60$ - 0.70; Carragher et al., 2018; Carragher et al., 2019). An a priori power analysis (G*Power; Faul, Erdfelder, Lang, \& Buchner, 2007) indicated that a sample of 33 participants was required to achieve $95 \%$ power to detect an effect of $d=0.65$ in a two-tailed one sample $t$-test with a conventional alpha of $\alpha=.05$. These parameters are consistent with the analytic approach used by Walker and Vul (2014) and Carragher and colleagues (2018; 2019; see Analysis below).

Sixty-six participants (54 females, $M_{\text {age }}=22.71, S D=6.77$ ) were randomly allocated to either Experiment 1a (moderate presentation time manipulation) or Experiment $1 \mathrm{~b}$ (extreme presentation time manipulation). Prior to analysis (Carragher et al., 2018), exclusion criteria were adopted to exclude all data from participants with a cheerleader effect score 
further than $3 S D$ from a single condition mean $(n=1)$ and from those who had incomplete data due to technical failure $(n=1)$. The final sample consisted of 32 participants in Experiment 1a (27 females, $\left.M_{\text {age }}=23.78, S D=8.78\right)$ and 32 participants in Experiment $1 \mathrm{~b}$ (26 females, $\left.M_{\text {age }}=21.78, S D=4.06\right)$.

The Social and Behavioural Research Ethics Committee of Flinders University approved the current research. All participants were recruited from Flinders University, gave written informed consent prior to testing, were debriefed upon completion, and received course credit or \$10AUD for their participation. Each testing session lasted between 15-20 minutes.

\section{Apparatus}

Both experiments were programmed using E Prime 2.0 software (Psychology Software Tools, Pittsburgh, PA; Schneider, Eschman, \& Zuccolotto, 2002), and were presented on a 22 " monitor $(1680 \times 1050 \mathrm{px})$ with a refresh rate of $60 \mathrm{~Hz}$. Stimuli were viewed at an approximate distance of $500 \mathrm{~mm}$. Individual portrait images were presented in the centre of the display $\left(70 \mathrm{~mm} \times 80 \mathrm{~mm} ; 8.00^{\circ} \times 9.15^{\circ}\right)$. Group images were created by presenting three individual portraits side by side $\left(210 \mathrm{~mm}\right.$ x $80 \mathrm{~mm} ; 23.72^{\circ}$ x $9.15^{\circ}$; Carragher et al., 2018; Carragher et al., 2019).

\section{Stimuli}

All face stimuli were selected from the stimulus set created by Carragher et al. (2018). Briefly, this stimulus set consists of female faces, which were collected by initially querying an online search engine with the term "bridesmaids" to find images of females in naturally assembled groups. The final stimulus set contains only individual portrait images, which were created by closely cropping the individual faces from these original group images. Carragher et al. (2018) reported that all faces in the stimulus set were female and showed happy 
expressions, and that most faces appeared to be between 20-40 years of age and of Caucasian ethnicity.

From this stimulus set, we randomly selected 99 faces to be the target faces that were presented twice; once in a group with two distractor faces, and once alone. Each observer only saw each target face in one group presentation time condition; however, the assignment of the target faces was counterbalanced between-participants, such that each face was seen in all presentation time conditions. Distractor faces were randomly selected to fill each group image from the remaining faces in the stimulus set. The position of the target and distractor faces in the group (i.e., left, centre, right) was pseudo-randomly assigned to ensure that target faces appeared with equal frequency in each position. The same face stimuli were used in Experiments 1a and 1b.

Both experiments were blocked by presentation time condition; however, the group and alone trial for each target image were presented within the same experimental block. Therefore, each block contained 33 group trials and 33 alone trials. In total, each participant completed 198 trials; 99 group trials [33 shorter condition (Experiment 1a: $1000 \mathrm{ms;}$ Experiment 1b: $300 \mathrm{~ms}$ ), 33 control condition (always $2000 \mathrm{~ms}$ ), 33 longer condition (Experiment 1a: 3000 ms; Experiment 1b: $7000 \mathrm{~ms}$ )], and 99 alone trials (2000 ms).

\section{Procedure}

Participants were asked to rate the attractiveness of each target face, which was eventually cued among the distractors in the group by a red frame that appeared around the border of the image. Participants completed six practice trials to familiarise themselves with the task. Group images were initially presented without the target identified (uncued duration). At this time, each face was surrounded by a black frame, and participants were encouraged to look at each face in the group. The duration of the uncued presentation varied depending on presentation time condition. Regardless of the uncued presentation duration, 
the red frame always appeared around the target face for an additional $1000 \mathrm{~ms}$ (cue), after which time all images disappeared from the screen (Carragher et al., 2018; Carragher et al., 2019; Walker \& Vul, 2014). During alone trials, the target face was presented in isolation for $1000 \mathrm{~ms}$ with a black frame, and then for an additional $1000 \mathrm{~ms}$ with a red frame, before disappearing from the screen (see Figure 1). Once all images had been removed from the display, participants gave their attractiveness rating for the target face via mouse-click along a visual analogue scale that ranged from "Very Unattractive" to "Very Attractive" $(0 \%$ $100 \%$; width $=192 \mathrm{~mm} ; 21.74^{\circ}$; Carragher et al., 2018).

a)

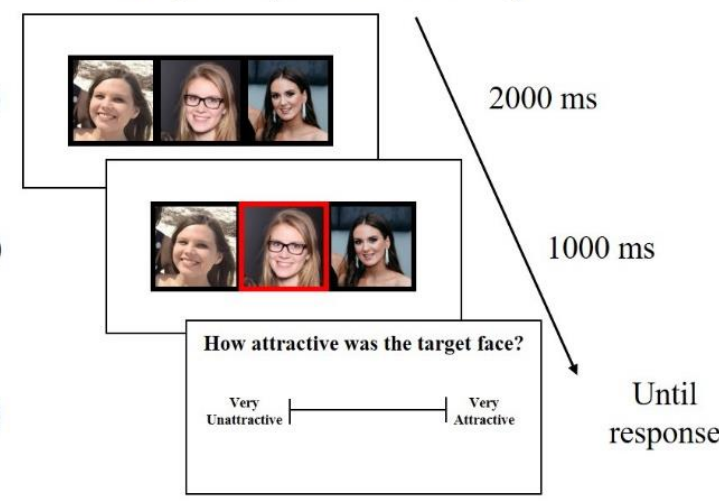

Alone Trial

d)

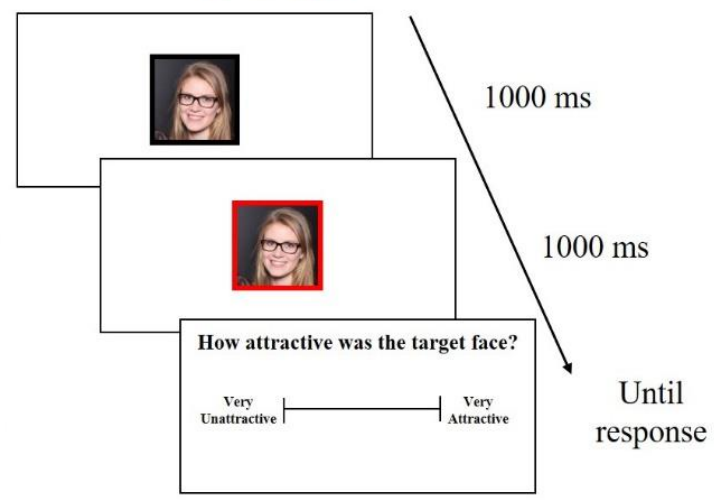

Figure 1. Examples of the group and alone trial procedures. Group Trial (Control Condition) $a$ ) the group is initially presented without the target cued $b$ ) the target is cued for $1000 \mathrm{~ms}$ [centre] c) an attractiveness rating is given along the visual analogue scale. Alone Trial $d$ ) the target is presented for $1000 \mathrm{~ms} e$ ) the target is cued for $1000 \mathrm{~ms} f$ ) an attractiveness rating is given along the visual analogue scale. [Due to copyright restrictions, the faces in this figure are representative of those in the stimulus set, but were not themselves shown in the experiment].

\section{Analysis}

We report the cheerleader effect as a change score, calculated by subtracting the attractiveness rating given to the target face when seen alone from the attractiveness rating given to the same target face when seen in a group. Therefore, a positive change score represents an increase in attractiveness in a group, whereas a negative score signals a decrease in attractiveness. Separate cheerleader effect measures were calculated for the three 
presentation time conditions in Experiments 1a and 1b. Consistent with the analytic strategy we have previously reported (Carragher et al., 2018; Carragher et al., 2019), we initially used separate one sample $t$-tests to determine whether the change in attractiveness was statistically significant in any of the group conditions (i.e., to first show whether the cheerleader effect occurred). We then conducted a mixed-model analysis of variance (ANOVA) to investigate whether the size of the cheerleader effects in the different group presentation time conditions differed from one another (i.e., to investigate whether presentation time modulated the cheerleader effect). Crucially, it is necessary to conduct the one sample $t$-tests before the ANOVA, because the ANOVA itself does not test whether the cheerleader effect actually occurred. Finally, we supplemented all conventional frequentist analyses with equivalent Bayesian analyses, to investigate whether any of our data provide evidence in favour of the null hypothesis (Wagenmakers et al., 2018). We adopted the classification scheme offered by Lee and Wagenmakers (2014) to interpret the significance of our Bayes factors. All analyses were conducted in JASP (Version 0.11.1; JASP Team, 2019).

\section{Data Availability}

All data reported in the current study are available in the Open Science Framework repository, [osf.io/3fhcp/]. A pre-print of this work is maintained on PsyArXiv [psyarxiv.com/nbc8e/].

\section{Results}

The target faces were randomly selected from the larger stimulus set. First, we examined how attractive these target faces were, using the attractiveness ratings given to the faces when they were seen alone. Because the same target faces were shown to participants in Experiment $1 \mathrm{a}$ and $1 \mathrm{~b}$, we averaged their ratings together to reduce variability in this first analysis. The average target face was perceived to be moderately attractive by all participants $(M=48.07 \%, S D=9.25, \operatorname{Min}=28.58 \%, \operatorname{Max}=72.61 \%)$, and a one-sample Kolmogorov- 
Smirnov normality test showed that the attractiveness ratings were normally distributed $(p>$ $.05)$.

A series of one sample $t$-tests showed that target faces were perceived to be significantly more attractive in each group condition in Experiment 1a and Experiment 1b, confirming that a statistically significant cheerleader effect occurred in every presentation time condition tested (see Table 1). We supplemented our frequentist analyses with equivalent Bayesian one samples $t$-tests. The data in Experiment 1a provide strong evidence for the cheerleader effect in the $1000 \mathrm{~ms}$ and $3000 \mathrm{~ms}$ presentation time conditions, and very strong evidence for the effect in the $2000 \mathrm{~ms}$ control condition. Similarly, there is very strong evidence for the cheerleader effect in the control condition of Experiment 1b, and moderateto-strong evidence for the effect in the $7000 \mathrm{~ms}$ condition, but only anecdotal evidence for the effect in the $300 \mathrm{~ms}$ condition.

\section{Table 1}

Frequentist and Bayesian one sample t-tests comparing cheerleader effect change scores in each presentation time condition with 0 , to determine statistically significant change in attractiveness.

\begin{tabular}{crrrrrrl}
\hline & $M$ & $S D$ & $t(31)$ & $95 \% C I$ & $p$ & $d$ & $\mathrm{BF}_{10}$ \\
\cline { 3 - 7 } Experiment 1a & & & & & & & \\
\cline { 5 - 8 } $1000 \mathrm{~ms}$ & $1.73 \%$ & 2.79 & 3.50 & $0.72,2.73$ & .001 & 0.62 & 23.76 \\
$2000 \mathrm{~ms}$ & $1.91 \%$ & 2.88 & 3.74 & $0.87,2.95$ & $<.001$ & 0.66 & 41.81 \\
$3000 \mathrm{~ms}$ & $1.24 \%$ & 2.24 & 3.13 & $0.43,2.05$ & .004 & 0.55 & 10.18 \\
\begin{tabular}{c} 
Experiment 1b \\
\hline $300 \mathrm{~ms}$
\end{tabular} & $1.33 \%$ & 3.47 & 2.16 & $0.08,2.58$ & .038 & 0.38 & 1.45 \\
$2000 \mathrm{~ms}$ & $1.65 \%$ & 2.47 & 3.77 & $0.76,2.53$ & $<.001$ & 0.67 & 45.55 \\
$7000 \mathrm{~ms}$ & $1.21 \%$ & 2.22 & 3.08 & $0.41,2.01$ & .004 & 0.54 & 9.02 \\
\hline
\end{tabular}

A mixed-model ANOVA with uncued presentation time (shorter, control, longer) as a within-participants factor, and experiment $(1 \mathrm{a}, 1 \mathrm{~b})$ as a between-participants factor, was used to investigate whether the duration of the uncued group presentation influenced the size of the cheerleader effect (see Figure 2). The main effects of uncued presentation time, $[F(2,124)=$ $\left.0.95, p=.391, \eta_{p}^{2}=.015\right]$, and experiment were non-significant, $\left[F(1,62)=0.22, p=.643, \eta_{p}^{2}\right.$ 
$=.003]$, as was the interaction between the two factors, $\left[F(2,124)=0.11, p=.899, \eta_{p}^{2}=\right.$ $.002]$.

To investigate whether the non-significant results from the ANOVA could be interpreted as providing support for the null-hypothesis, we conducted a Bayesian mixedmodel ANOVA. The main effects of presentation time $\left(\mathrm{BF}_{10}=0.13\right)$ and experiment $\left(\mathrm{BF}_{10}=\right.$ 0.26) provided moderate support for the null hypothesis (likelihood ratios of 8.14 and 3.95 respectively), while the interaction between the two factors $\left(\mathrm{BF}_{10}<0.01\right)$ provided extreme evidence for the null hypothesis (likelihood ratio $=311.17$ ).

Finally, to address our specific hypothesis for Experiment 1a, a planned pairedsamples $t$-test was used to investigate whether the cheerleader effect was significantly larger in the 2000 ms condition than the 3000 ms condition, which would indicate that the known presentation time discrepancy contributes to the cheerleader effect. The size of the cheerleader effect did not differ significantly between the $2000 \mathrm{~ms}$ and $3000 \mathrm{~ms}$ presentation time conditions, $[t(31)=1.17,95 \% C I(-0.50,1.82), p=.252, d=0.21]$, and a Bayesian paired samples $t$-test indicated that these data provide anecdotal support for the null hypothesis $\left(\mathrm{BF}_{10}=0.35\right.$, likelihood ratio $\left.=2.84\right)$. Taken together, our findings demonstrate that uncued presentation time does not modulate the size of the cheerleader effect. 


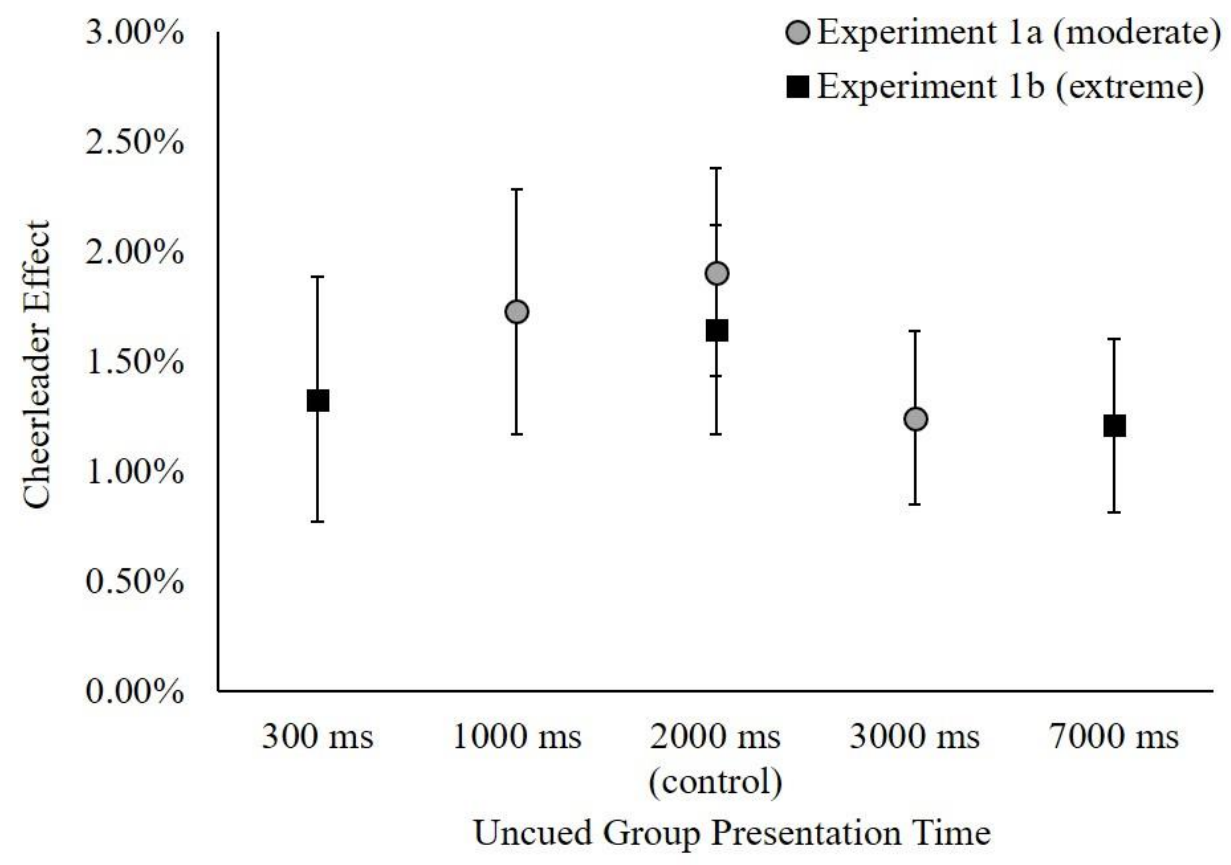

Figure 2. The cheerleader effect for each presentation time condition in Experiment 1a and Experiment $1 \mathrm{~b}$. The cheerleader effect is statistically significant in each condition (see Table 1). Error bars show the standard error of the mean.

\section{Discussion}

The cheerleader effect occurred in each condition tested, regardless of the duration of uncued presentation. Crucially, there was no significant difference between the $2000 \mathrm{~ms}$ and $3000 \mathrm{~ms}$ presentation time conditions in Experiment 1a, demonstrating that the discrepancy between the presentation time of the target in the group and alone conditions in previous paradigms does not contribute to the size of the cheerleader effect. Furthermore, the cheerleader effect occurred both when the uncued presentation of the group was extremely brief, and when the available viewing time of the target far exceeded that of the alone presentation time condition. Taken together, our results suggest that presentation time has very little influence on the size of the cheerleader effect. These results demonstrate that presentation time is not a contributing factor to the two-mechanism frameworks of the cheerleader effect proposed by Carragher et al. (2019) and Ying et al. (2019). 
These findings are consistent with those recently reported by Ying et al. (2019) and McDowell and Starratt (2019), who both incidentally manipulated the presentation time of the group image in their cheerleader effect paradigms. Ying et al. (2019) found significant cheerleader effects in a psychophysical paradigm, wherein the target face was presented for $1000 \mathrm{~ms}$ in a group condition without any uncued encoding time, which matched the presentation time available in the alone condition (1000 ms). Moreover, because the location of the target face was always known in advance, the observers should have fixated the target for the entire presentation duration (Ying et al., 2019). On the other hand, McDowell and Starratt (2019) reported a significant cheerleader effect in a paradigm that required participants to rate the attractiveness of all 6 faces in a group image that was presented for 45 seconds, and then again when each face was presented alone for 7 seconds. When considered alongside the results of the current study, these findings further suggest that the cheerleader effect does not require highly precise timing between the group and alone conditions to be observed.

Although our primary aim was to investigate whether presentation time might inadvertently contribute to the size of the cheerleader effect, these data can also be used to reevaluate the other proposed causes of the effect. Notably, our results are inconsistent with the hierarchical encoding mechanism (Walker \& Vul, 2014). Hierarchical encoding relies on ensemble coding, a process that serves to rapidly establish gist representations of complex visual scenes, which are no longer necessary once the observer is given enough time to encode each face in the group individually (Alvarez, 2011; Li et al., 2016; Neumann, Ng, Rhodes, \& Palermo, 2017). In ensemble coding tasks, observers become more accurate in their judgments of the individual faces in the group, and less biased by the ensemble average, as the presentation duration of the group image increases beyond $1500 \mathrm{~ms}$ (Li et al., 2016; Neumann et al., 2017). Notably, we found a significant cheerleader effect when target faces 
were presented in group images for $7000 \mathrm{~ms}$; a duration that far exceeds that which is typical for ensemble coding (Li et al., 2016; Neumann et al., 2017). These results indirectly suggest that ensemble coding, a pre-requisite step for the hierarchical encoding mechanism (Walker \& Vul, 2014), is not necessary for the cheerleader effect to occur. Consistent with previous research (Carragher et al., 2019; Ying et al., 2019), our current findings further indicate that the cheerleader effect is not caused by hierarchical encoding (Walker \& Vul, 2014).

The results of the current study are less informative about the two-mechanism accounts of the cheerleader effect suggested by Carragher et al. (2019) and Ying et al. (2019). Both authors suggested that an initial increase in attractiveness might occur due to a mechanism of social inference (Carragher et al., 2019; Ying et al., 2019). Ying et al. (2019) propose that the second mechanism of the cheerleader effect is one of contrast (see also van Osch et al., 2015), whereby the magnitude of attractiveness change depends on the attractiveness of the target face relative to the distractor faces in the group. Since neither the social inference nor contrast mechanisms have defined presentation time dependant components, the results of the current study do not shed light on their possible roles in the cheerleader effect. Our results are more informative for narrowing down the additional mechanisms considered by Carragher et al. (2019). To the extent that Carragher et al. (2019) leave open the possibility that the second mechanism could be hierarchical encoding, for the reasons described above, this possibility now appears unlikely. Our current findings also make a mechanism of selective attention toward the most attractive faces in the group less likely, since the effects of selective attention would be most pronounced under the strictest time constraints (Carragher, 2018; van Osch et al., 2015). In light of the evidence presented here, we suggest that Ying et al.'s (2019) two-mechanism account of the cheerleader effect appears to be the most consistent with the data collected to date. Future research should be directed toward investigating the nature of the relatively abstract "social inference" 
mechanism (Carragher et al., 2019; Ying et al., 2019), as well as the role of contrast between the attractiveness of the group members in the cheerleader effect (Ying et al., 2019).

Taken together, our findings raise some interesting questions about the possible consequences of the cheerleader effect in the real world. Considering that the same face was perceived to be more attractive in a group that had been shown for $7000 \mathrm{~ms}$, is it possible that the same face will always be perceived to be more attractive in a group than alone, regardless of how long the group is viewed? Attractiveness has long been known to confer benefits to individuals as a result of the "what is beautiful is good" stereotype (Dion, Berscheid, \& Walster, 1972; Eagly, Ashmore, Makhijani, \& Longo, 1991; Langlois et al., 2000). Attractive individuals go on more dates (Langlois et al., 2000), receive more job offers (Gilmore, Beehr, \& Love, 1986), and even get more votes in elections than unattractive individuals (Stockemer \& Praino, 2015). If the cheerleader effect occurs regardless of how long an individual is seen in a group, is it possible that one might go on more dates simply by posting more group photographs to online dating websites? Or would a candidate receive more votes in an election by including more pictures of themselves posing among groups of their constituents in their campaign advertisements? Of course, it has yet to be seen whether such a small increase in attractiveness (1.5\%-2.0\%) is enough to have an observable effect on these realworld outcomes. Future research that investigates the consequences of the cheerleader effect in these real-world situations will be necessary to address these questions.

The results of the current study demonstrate that the cheerleader effect is not the result of a methodological presentation time discrepancy (Carragher, 2018; Walker \& Vul, 2014), and that presentation time is not likely a contributing factor to the size of the typical cheerleader effect (Carragher et al., 2019). Despite this step forward, future research is still needed to identify the underlying cause of the cheerleader effect. Nonetheless, when taken together, these results are consistent with the emerging research to suggest that the 
cheerleader effect is a remarkably robust effect that can be replicated under a number of different experimental parameters. 


\section{Acknowledgements:}

While conducting this research, author DJC was supported by an Australian Government Research Training Scholarship.

\section{Competing Interests:}

The authors have no competing interests to declare.

\section{Financial Disclosure:}

This research did not receive any specific grant from funding agencies in the public, commercial, or not-for-profit sectors. 


\section{References}

Alvarez, G. A. (2011). Representing multiple objects as an ensemble enhances visual cognition. Trends in Cognitive Sciences, 15(3), 122-131. doi:10.1016/j.tics.2011.01.003

Ariely, D. (2001). Seeing sets: Representation by statistical properties. Psychological Science, 12(2), 157-162. doi:10.1111/1467-9280.00327

Brady, T. F., \& Alvarez, G. A. (2011). Hierarchical encoding in visual working memory: Ensemble statistics bias memory for individual items. Psychological Science, 22(3), 384-392. doi:10.1177/0956797610397956

Carragher, D. J. (2018). Social Perception in Group Scenes: Social context modulates perceptions of facial attractiveness. (PhD Thesis), Flinders University, Unpublished. doi: 10.13140/RG.2.2.20048.56326

Carragher, D. J., Lawrence, B. J., Thomas, N. A., \& Nicholls, M. E. R. (2018). Visuospatial asymmetries do not modulate the cheerleader effect. Scientific Reports, 8(1), 2548. doi:10.1038/s41598-018-20784-5

Carragher, D. J., Thomas, N. A., Gwinn, O. S., \& Nicholls, M. E. R. (2019). Limited evidence of hierarchical encoding in the cheerleader effect. Scientific Reports, 9(1), 9329. doi:10.1038/s41598-019-45789-6

Crouzet, S. M., Kirchner, H., \& Thorpe, S. J. (2010). Fast saccades toward faces: Face detection in just $100 \mathrm{~ms}$. Journal of Vision, 10(4), 16-16. doi:10.1167/10.4.16

de Fockert, J., \& Wolfenstein, C. (2009). Rapid extraction of mean identity from sets of faces. The Quarterly Journal of Experimental Psychology, 62(9), 1716-1722. doi:10.1080/17470210902811249

Dion, K., Berscheid, E., \& Walster, E. (1972). What is beautiful is good. Journal of Personality and Social Psychology, 24(3), 285-290. doi:10.1037/h0033731

Eagly, A. H., Ashmore, R. D., Makhijani, M. G., \& Longo, L. C. (1991). What is beautiful is good, but...: A meta-analytic review of research on the physical attractiveness stereotype. Psychological Bulletin, 110(1), 109-128. doi:10.1037//00332909.110.1.109

Faul, F., Erdfelder, E., Lang, A.-G., \& Buchner, A. (2007). G* Power 3: A flexible statistical power analysis program for the social, behavioral, and biomedical sciences. Behavior Research Methods, 39(2), 175-191. doi:10.3758/bf03193146

Gerger, G., Forster, M., \& Leder, H. (2017). It felt fluent but I did not like it: Fluency effects in faces versus patterns. The Quarterly Journal of Experimental Psychology, 70(4), 637-648. doi:10.1080/17470218.2016.1145705

Gilmore, D. C., Beehr, T. A., \& Love, K. G. (1986). Effects of applicant sex, applicant physical attractiveness, type of rater and type of job on interview decisions. Journal of Occupational Psychology, 59(2), 103-109. doi:10.1111/j.2044-8325.1986.tb00217.x

Griffiths, S., Rhodes, G., Jeffery, L., Palermo, R., \& Neumann, M. F. (2018). The average facial expression of a crowd influences impressions of individual expressions. Journal of Experimental Psychology: Human Perception and Performance, 44(2), 311-319. doi:10.1037/xhp0000446

Haberman, J., \& Whitney, D. (2007). Rapid extraction of mean emotion and gender from sets of faces. Current Biology, 17(17), R751-R753. doi:10.1016/j.cub.2007.06.039

Haberman, J., \& Whitney, D. (2009). Seeing the mean: Ensemble coding for sets of faces. Journal of Experimental Psychology: Human Perception and Performance, 35(3), 718-734. doi:10.1037/a0013899

JASP Team. (2019). JASP (Version 0.11.1)[Computer software]. 
Langlois, J. H., Kalakanis, L., Rubenstein, A. J., Larson, A., Hallam, M., \& Smoot, M. (2000). Maxims or myths of beauty? A meta-analytic and theoretical review. Psychological Bulletin, 126(3), 390-423. doi:10.1037//0033-2909.126.3.390

Langlois, J. H., \& Roggman, L. A. (1990). Attractive faces are only average. Psychological Science, 1(2), 115-121. doi:10.1111/j.1467-9280.1990.tb00079.x

Lee, M. D., \& Wagenmakers, E.-J. (2014). Bayesian cognitive modeling: A practical course: Cambridge university press.

Lew, T. F., \& Vul, E. (2015). Ensemble clustering in visual working memory biases location memories and reduces the Weber noise of relative positions. Journal of Vision, 15(4), 10-10. doi:10.1167/15.4.10

Li, H., Ji, L., Tong, K., Ren, N., Chen, W., Liu, C. H., \& Fu, X. (2016). Processing of individual items during ensemble coding of facial expressions. Frontiers in Psychology, 7, 1332. doi:10.3389/fpsyg.2016.01332

McDowell, J., \& Starratt, V. G. (2019). Experimental examination and extension of the cheerleader effect. Personality and Individual Differences, 147, 245-249. doi:10.1016/j.paid.2019.05.004

Neumann, M. F., Ng, R., Rhodes, G., \& Palermo, R. (2017). Ensemble coding of face identity is not independent of the coding of individual identity. The Quarterly Journal of Experimental Psychology, 71(6), 1357-1366. doi:10.1080/17470218.2017.1318409

Neumann, M. F., Schweinberger, S. R., \& Burton, A. M. (2013). Viewers extract mean and individual identity from sets of famous faces. Cognition, 128(1), 56-63. doi:10.1016/j.cognition.2013.03.006

Rashidi, M., Pazhoohi, F., \& Hosseinchari, M. (2012). Effect of facial stimuli exposure time on evaluation of facial attractiveness. Australian Journal of Psychology, 64(3), 164168. doi:10.1111/j.0004-9530.2011.00050.x

Rayner, K. (1998). Eye movements in reading and information processing: 20 years of research. Psychological Bulletin, 124(3), 372-422. doi:10.1037//0033-2909.124.3.372

Rhodes, G. (2006). The evolutionary psychology of facial beauty. Annual Review of Psychology, 57(1), 199-226. doi:10.1146/annurev.psych.57.102904.190208

Rodeheffer, C. D., Proffitt Leyva, R. P., \& Hill, S. E. (2016). Attractive female romantic partners provide a proxy for unobservable male qualities: The when and why behind human female mate choice copying. Evolutionary Psychology, 14(2), 1474704916652144. doi:10.1177/1474704916652144

Saegusa, C., \& Watanabe, K. (2016). Judgments of facial attractiveness as a combination of facial parts information over time: Social and aesthetic factors. Journal of Experimental Psychology: Human Perception and Performance, 42(2), 173. doi:10.1037/xhp0000149

Schneider, W., Eschman, A., \& Zuccolotto, A. (2002). E-prime (version 2.0). Computer software and manual]. Pittsburgh, PA: Psychology Software Tools Inc.

Stockemer, D., \& Praino, R. (2015). Blinded by beauty? Physical attractiveness and candidate selection in the US House of Representatives. Social Science Quarterly, 96(2), 430443. doi:10.1111/ssqu. 12155

van Osch, Y., Blanken, I., Meijs, M. H., \& van Wolferen, J. (2015). A group's physical attractiveness is greater than the average attractiveness of its members: The Group Attractiveness Effect. Personality and Social Psychology Bulletin, 41(4), 559-574. doi:10.1177/0146167215572799

Wagenmakers, E.-J., Marsman, M., Jamil, T., Ly, A., Verhagen, J., Love, J., . . Epskamp, S. (2018). Bayesian inference for psychology. Part I: Theoretical advantages and practical ramifications. Psychonomic Bulletin \& Review, 25(1), 35-57. doi:10.3758/s13423-017-1343-3 
Walker, D., \& Vul, E. (2014). Hierarchical encoding makes individuals in a group seem more attractive. Psychological Science, 25(1), 230-235. doi:10.1177/0956797613497969

Wedell, D. H., Parducci, A., \& Geiselman, R. E. (1987). A formal analysis of ratings of physical attractiveness: Successive contrast and simultaneous assimilation. Journal of Experimental Social Psychology, 23(3), 230-249. doi:10.1016/0022-1031(87)90034-5

Whitney, D., \& Yamanashi Leib, A. (2018). Ensemble perception. Annual Review of Psychology, 69(1), 105-129. doi:10.1146/annurev-psych-010416-044232

Willis, J., \& Todorov, A. (2006). First impressions: Making up your mind after a 100-ms exposure to a face. Psychological Science, 17(7), 592-598. doi:10.1111/j.14679280.2006.01750.x

Ying, H., Burns, E., Lin, X., \& Xu, H. (2019). Ensemble statistics shape face adaptation and the cheerleader effect. Journal of Experimental Psychology: General, 148(3), 421436. doi:10.1037/xge0000564 